PREPRINT -- NOT PEER REVIEWED

\title{
Perception of changes in bar velocity as a resistance training monitoring tool for athletes
}

Amit Lazarus ${ }^{1} \uparrow$, Israel Halperin ${ }^{2,3} \dagger^{*}$, Gal Yosef Vaknin ${ }^{4}$, and Antonio Dello Iacono 5

Affiliations:

${ }^{1}$ Faculty of Social Sciences, Department of Sociology and Anthropology, Tel Aviv University, Tel Aviv-Yafo, Israel

${ }^{2}$ School of Public Health, Sackler Faculty of Medicine, Tel Aviv University, Tel AvivYafo, Israel

${ }^{3}$ Sylvan Adams Sports Institute, Tel Aviv University, Tel Aviv-Yafo, Israel

${ }^{4}$ Sektzia Nes Tziona football club, Nes Tziona, Israel

${ }^{5}$ School of Health and Life Sciences, University of the West of Scotland, Hamilton, United Kingdom,

$\uparrow$ Amit Lazarus and Israel Halperin are joint first authors as they have contributed equally to this manuscript.

*Corresponding author: Israel Halperin, School of Public Health, Sackler Faculty of Medicine, Tel Aviv University, Tel Aviv-Yafo, Israel.

Phone: +972-523-469-590

Email: ihalperin@tauex.tau.ac.il

Author agreement statement: We the authors agree to the sharing of this preprint onSportR $\chi$ iv.

Cite as: Lazarus, A., Halperin, I., Vaknin, G. Y., \& Dello Iacono, A. (2020, December 19). Perception of changes in bar velocity as a resistance training monitoring tool for athletes. Pre-print DOI: https://doi.org/10.31236/osf.io/qstkp 


\begin{abstract}
Purpose: To investigate if perception of changes in bar velocity (PCV) can be used as a substitute for velocity tracking devices commonly used to monitor resistance-exercises. Methods: Twenty-one professional male soccer athletes $(21.1 \pm 4.3$ years $)$ first went through a load-power profile assessment to determine their optimal power load in the backsquat. In the next three experimental sessions, athletes completed four sets of six repetitions loaded with optimal power load. Starting from the second repetition, athletes reported their PCV of each repetition as a percentage of the first repetition. Accuracy of PCV was calculated as the absolute difference between PCV and the actual percentage change from the first repetition in bar velocity measured with a linear-encoder. The second and fourth sessions served as the pre- and post-intervention sessions, in which athletes received no feedback about their PCV accuracy. The third session served as the intervention, in which athletes received verbal and visual feedback about their PCV accuracy levels after each set. Results: The estimated accuracy of PCV decreased from an average error of $7 \%$ in the preintervention to an average error of $4.7 \%$ in the post-intervention session ( $95 \%$ confidence levels of difference: 1.5, 3.0). Conclusion: Athletes with velocity based training experience begin with a reasonable PCV accuracy rates which can be meaningfully improved after a single feedback session. When velocity tracking devices are impractical or absent, PCV can be implemented as a resistance training monitoring tool.
\end{abstract}

\title{
Introduction
}

Velocity-based training (VBT) is a method used to prescribe and monitor resistance training programs based on repetition velocity outputs during different exercises. ${ }^{1-4}$ VBT has a number of benefits. First, the velocity loss observed across repetitions provides an indication of neuromuscular fatigue ${ }^{5}$ and the number of repetitions left before one reaches task-failure. ${ }^{6}$ Second, a number of longitudinal studies found that terminating sets at certain velocity loss thresholds (e.g., 5-30\% relative to first repetition) can induce a range of neuromuscular adaptations. ${ }^{7-9}$ These benefits allow to individualize and thus optimize the training process by adjusting the number of repetitions performed per exercise. However, 
in order to implement VBT methodologies, velocity measuring devices are required (e.g., linear position transducers $\left[\mathrm{LPT}^{10}\right]$ ). While the costs of these devices have decreased in recent years, they are not affordable to many. ${ }^{11}$ Furthermore, using these devices with large groups of athletes can be a challenging task. Hence, in cases that velocity measuring devices are inaccessible or impractical, trainee's perception of movement velocity, or changes in movement velocity, can be used as a possible substitute. Assuming acceptable accuracy levels, applying VBT methodologies based on trainee's perception can be advantageous given its simple and free to use.

Recently, Sindiani et al. ${ }^{12}$ examined the accuracy levels of trainee's perception of changes in bar velocity (PCV) in comparison to actual bar velocity (ACV) measured with an LPT. Twenty resistance-trained subjects completed three sessions composed of four sets of eight repetitions in the squat and bench-press exercises. In two sessions, subjects lifted loads corresponding to $60 \%$ of one Repetition-Maximum (1RM), and in one session the loads corresponded to $70 \%$ of 1RM. Starting from the second repetition of each set, subjects rated their PCV as a percentage of the first repetition, which was preset to be $100 \%$ (i.e., the reference point), irrespective if it was the fastest repetition or not. To illustrate, if the third repetition was perceived to be $10 \%$ slower than the first repetition, then the person should have rated it as $90 \%$. Using this approach, two types of errors were analyzed. First, the absolute error, defined as the difference between PCV and the actual changes in bar velocity (ACV). Second, the directional error, defined as the likelihood of over- or underestimating ACV.

Sindiani et al. ${ }^{12}$ observed three key findings. First, across sessions and sets, the absolute error was $\sim 5.8$ percentage-points in both exercises in the second repetition and increased to 13.2 and 16.7 percentage-points by the eighth repetition in the bench-press and squat, respectively. The increments in error followed a linear pattern, increasing by 1.2 and 1.8, percentage-points with consecutive repetitions in the bench-press and squat, respectively. Second, as a whole, subjects improved their accuracy in the second 60\%1RM session compared to the first by $\sim 1.7$ percentage-points in both exercises. Finally, subjects were 4.2 times more likely to underestimate velocity in the squat compared to the bench-press. Based on these results, the authors proposed a number of future research directions, two of 
which are relevant to the present study. First, there is a need to investigate PCV accuracy levels among different populations. Specifically, while subjects in Sindiani's study were experienced in resistance training, none were accustomed to VBT. It may be that prior experience with VBT is associated with reduced absolute and directional errors. Second, there is a need to investigate to what extent accuracy levels can be improved. The fact that subjects reduced the absolute error in the second 60\%1RM session in the study by Sindiani et al. ${ }^{12}$ suggests that practice can enhance accuracy levels. Importantly, the observed improvement occurred despite the fact that subjects were not provided with feedback on $\mathrm{ACV}$. It is thus of interest to examine to what extent accuracy levels can be improved when subjects are presented with feedback on ACV.

Therefore, the purpose of the present study was to complement and expand upon Sindiani's work by: (i) investigating PCV accuracy rates during the barbell squat exercise among competitive athletes experienced with VBT; (ii) Investigate if, and to what extent, PCV accuracy rates can be improved among this cohort when receiving augmented feedback concerning the extent and direction of error.

\section{Materials and Methods}

\section{Participants}

A convenience sample of 21 male national-level professional soccer players volunteered to participate in the study (age: $21.1 \pm 4.3$ years; height: $178.3 \pm 5.8 \mathrm{~cm}$; body mass: $72.9 \pm 6.2$ $\mathrm{kg}$; optimum power load (OPL): $51.7 \pm 6.7 \%$ of 1RM). Whereas all 21 completed the first two sessions, only 16 completed the final session due to the COVID-19 outbreak. All athletes had at least 12 years (range: 12-27) of soccer training and three years (range: 3-9) of resistance training experience. All athletes also had at least one year (range: 1-7) of resistance training experience involving VBT methodologies. Written informed consent was obtained after athletes received an oral explanation of the purpose, benefits, and potential risks of the study. All procedures were conducted in accordance with the Helsinki Declaration and approved by the Institution's Ethics Committee. 


\section{Procedure}

A pre-post within-subject design was used to investigate (i) the accuracy levels of PCV in the barbell squat exercise performed in a Smith-Machine (Technogym Equipment, Barcelona, Spain) among professional soccer players familiar with VBT; (ii) examine if, and to what extent, PCV accuracy can be improved among this cohort when receiving augmented feedback on the extent and direction of their errors. Athletes reported to the laboratory on four occasions separated by three to six days. In the first session, participants were assessed to determine the individual OPL in the barbell squat and were familiarized with the experimental procedures. In the three subsequent experimental sessions, participants followed the exact same procedures, consisting of four sets of six repetitions of squats using the individualized OPL, with three minutes of rest between the sets. Starting from the second repetition, participants verbally reported their PCV of each repetition which was recorded via a tie-microphone attached to their shirts. ACV was measured with an LPT. While the second and forth sessions were identical, serving as the pre and postintervention session, in the third session athletes received both verbal and visual feedback concerning their accuracy after each set.

\section{RM tests and familiarization (session 1)}

Anthropometric measurements were taken and followed by an assessment of the required squat depth corresponding to a $90^{\circ}$ knee angle measured with a hand-held goniometer. To ensure similar depth across sessions, a box with adjustable height was placed underneath the participants to which they were required to gently squat onto. Athletes then performed a structured warm-up protocol consisting of dynamic stretching and calisthenics, followed by an individualized five-minutes warm-up. This warm-up protocol was identical in all sessions. Thereafter, athletes completed a load-power profile assessment to determine the individual OPL in the barbell squat using a Smith-machine. The OPLs were determined following the protocol described by Loturco et al. ${ }^{13}$ and identified as the absolute load resulting in the highest mean propulsive power values measured during progressively loaded trials. Briefly, the protocol consisted of consecutive sets of squats starting with $36 \mathrm{~kg}$ and $10-20 \mathrm{~kg}$ increments per set. Athletes performed three repetitions at maximum velocity 
for each progressive load until a clear decrement (observed over at least two loads) in power output was detected. This equated to approximately 5 testing sets. Two to three minutes of rest were provided between attempts. The individual OPLs were calculated via a dedicated software (Chronojump, Barcelona, Spain), and then used for the three experimental sessions.

Following the OPL assessment, participants were familiarized with the procedure of the experimental sessions by performing two sets of 8-12 repetitions with an unloaded barbell. Specifically, athletes were asked to verbally report their PCV starting from the second repetition and onward as a percent of the first repetition. That is, the first repetition was always considered $100 \%$ (i.e., the reference point) irrespective if the actual velocity resulted in the fastest repetition or not. Then, starting from the second repetition, participants reported percent values in relation to the first repetition. Participants were asked to execute the concentric phase of each repetition as fast as possible. After each completed repetition, participants were asked to verbally state their PCV.

\section{Experimental sessions (sessions 2-4)}

Following a standard warm-up, athletes completed a squat specific warm-up consisting of sets with progressive loads until reaching their respective OPL. After two minutes of rest, athletes completed four sets of six repetitions with the OPL in all three sessions. Whereas the second and forth session were identical and served as the pre- and post-intervention sessions, in which no velocity feedback was provided, in the third session athletes received augmented feedback after each set concerning their accuracy rates. Specifically, within $\sim 30$ seconds after each set the experimenter prepared an Excel graph illustrating both PCV and AVC velocity data points of each repetition (Figure 1). The experimenter showed the graph to each athlete during the rest period between sets and pointed out the extent and direction of the errors. These graphs were subsequently sent to each athlete and also used during a short, five-minute face-to-face meeting between the third and the fourth session, in which the experimenter went over the graphs with each athlete, highlighting their common errors. All sessions were performed at the same facility, ran by the same experimenter at 
approximately the same time of the day ( \pm 2 hours). Athletes were asked to refrain from intense training $24 \mathrm{~h}$ prior to testing days and to avoid muscular fatigue and soreness.

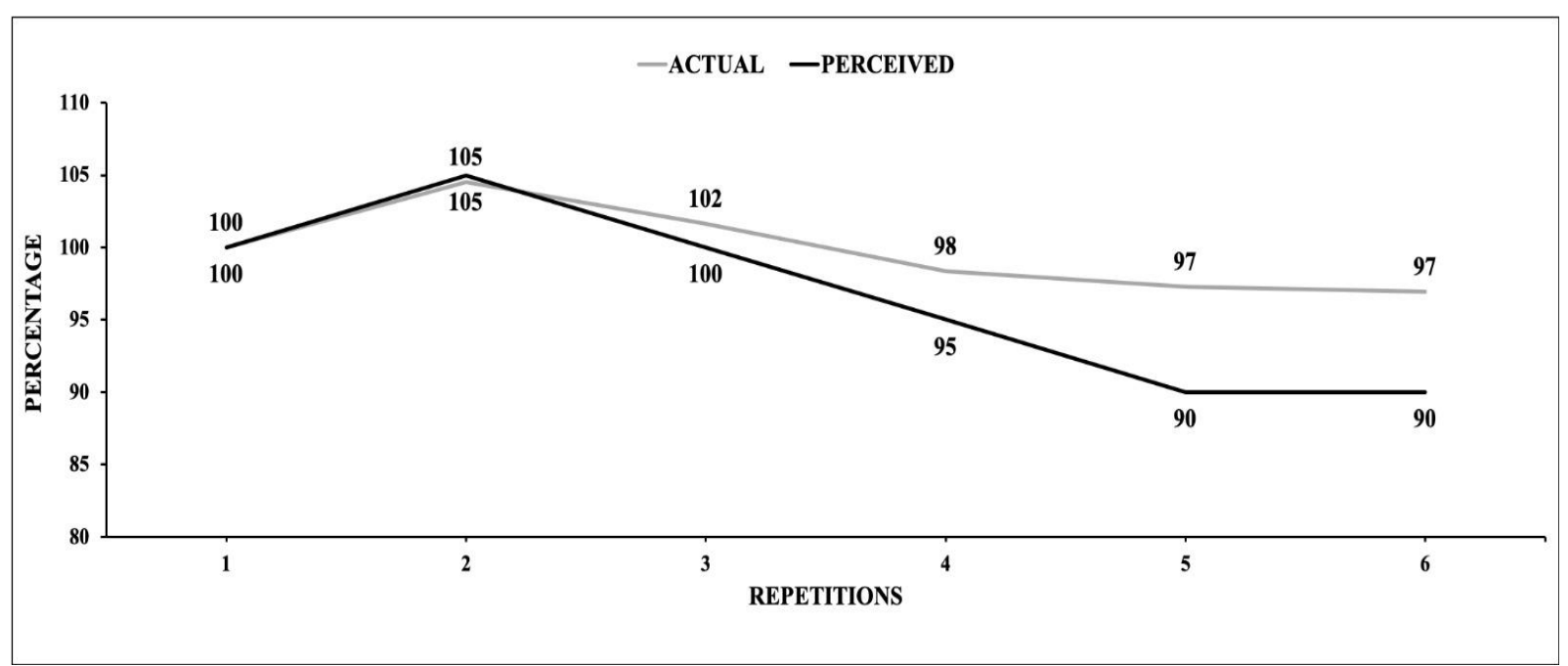

Figure 1. An example of the individualized graph presented to the athletes as feedback between sets in the intervention session.

\section{Bar velocity data collection}

The mean propulsive velocity of bar movement during the concentric phase for each repetition was collected. For this purpose, an LPT (Chronojump, Barcelona, Spain) sampling at $1000 \mathrm{~Hz}$, was fixed to the bar of the Smith machine at a perpendicular angle to the floor, and the commercial software provided by the manufacturer in conjunction with the device, was used to record the bar velocity outcomes. According to the software specifications, instantaneous velocity was smoothed with a fourth-order low-pass Butterworth filter, with a cut-off frequency of $10 \mathrm{~Hz}$.

\section{Statistical analysis}

We descriptively inspected the data points by plotting the raw percentage point differences between the PCV and ACV in all repetitions, relative to the first repetition. To inferentially analyze the absolute differences between PCV and ACV we used the following within subject fixed effects model:

$|P C V-A C V|_{i t}=\beta_{0}+\beta_{1}$ session $_{i t}+\beta_{2-4}$ set $_{i t}+\beta_{5}$ repetition $_{i t}+\beta_{k} z_{i t}+a_{i}+\varepsilon_{i t}$

where inaccuracy scores it $_{\text {it }}$ is the percentage point difference between PCV and ACV of subject $i$ at repetition $t ; \beta_{\mathrm{k}}$ represents the 4 coefficients of the two-way interactions between 
session (post-intervention=1) on the one hand and sets and repetitions on the other. Finally, $a_{i}$ is a subject specific deviation from the grand mean of the inaccuracy scores.

In contrast to Sindiani et al.'s study ${ }^{12}$ in which an analysis of the propensity of a unidirectional error (i.e., either under- or over-estimating the bar velocity) was reported in the main text, in the current study we decided to present this analysis as supplementary material because we did not find any theoretical or practical value of this analysis to the field (see online supplement).

\section{Results}

\section{Descriptive statistics}

Figure 2 depicts the absolute data points across sessions and repetitions. As seen in panel A (pre-intervention-absolute), both the average absolute error and the variance increases as repetitions progress. In contrast, after receiving the augmented feedback, in the postintervention (panel B) we note that the extent of error and its variance remained stable throughout the set.

\section{Extent of error}

Controlling for sets and repetitions, the absolute error was 2.3 percentage points higher in the pre- compared to the post-intervention session $(\mathrm{p}<0.001)$ (Table 1 and Figure 3). Controlling for sessions and repetitions, we observed no meaningful differences between sets $(\mathrm{p}>0.134,0.21<\mathrm{b}<0.76$ percentage points), nor an interaction between sets and sessions $(\mathrm{p}>0.165,-0.65<\mathrm{b}<1.41$ percentage points). In contrast, we found a significant effect of repetition, so that each passing repetition within a set added 0.45 percentage points to the absolute error. Moreover, the interaction of repetitions and session was significant $(b=-0.73, p=0.004)$. Specifically, while the effect of repetition was highly substantial and significant in the pre-intervention session $(b=0.76, p<0.001)$, it was inconsequential in the post-intervention session $(b=0.03, p=0.874)$. It therefore follows that differences in the observed error between the sessions grew larger as the number of repetitions progressed. For instance, in the first set the gap between the sessions in repetition two was nonsignificant at 1.4 percentage points $(\mathrm{p}=0.126)$ but it grew to a significant 4.3 percentage points by the repetition $6(\mathrm{p}<0.001)$. 
Table 1. Fixed effects (within subjects) linear regression of extent of error in velocity

\begin{tabular}{|c|c|c|c|c|}
\hline \multirow{3}{*}{$\begin{array}{l}\text { Fixed } \\
\text { Constant }\end{array}$} & \multicolumn{2}{|c|}{ Model 1} & \multicolumn{2}{|c|}{ Model 2} \\
\hline & $\underline{b}$ & $\underline{\text { S.E }}$ & $\underline{b}$ & $\underline{\text { S.E }}$ \\
\hline & $5.75 * * *$ & 0.47 & $5.34 * * *$ & 0.58 \\
\hline Post [Pre] & $-2.30 * * *$ & 0.38 & -1.36 & 0.89 \\
\hline [Set 1] & & & & \\
\hline Set 2 & 0.76 & 0.50 & 0.14 & 0.66 \\
\hline Set 3 & 0.20 & 0.50 & -0.37 & 0.66 \\
\hline Set 4 & 0.26 & 0.50 & 0.54 & 0.66 \\
\hline Repetition & $0.44 * * *$ & 0.12 & $0.76 * * *$ & 0.16 \\
\hline Post*set 2 & & & 1.41 & 1.01 \\
\hline Post*set 3 & & & 1.35 & 1.01 \\
\hline Post*set 4 & & & -0.64 & 1.01 \\
\hline Post*Repetition & & & $-0.733 * *$ & 0.25 \\
\hline $\begin{array}{l}\mathrm{N} \text { (within } 21 \\
\text { subjects) }\end{array}$ & & & 73 & \\
\hline
\end{tabular}

$* \mathrm{p}<0.05 ; * * \mathrm{p}<0.01 ; * * * \mathrm{p}<0.001$
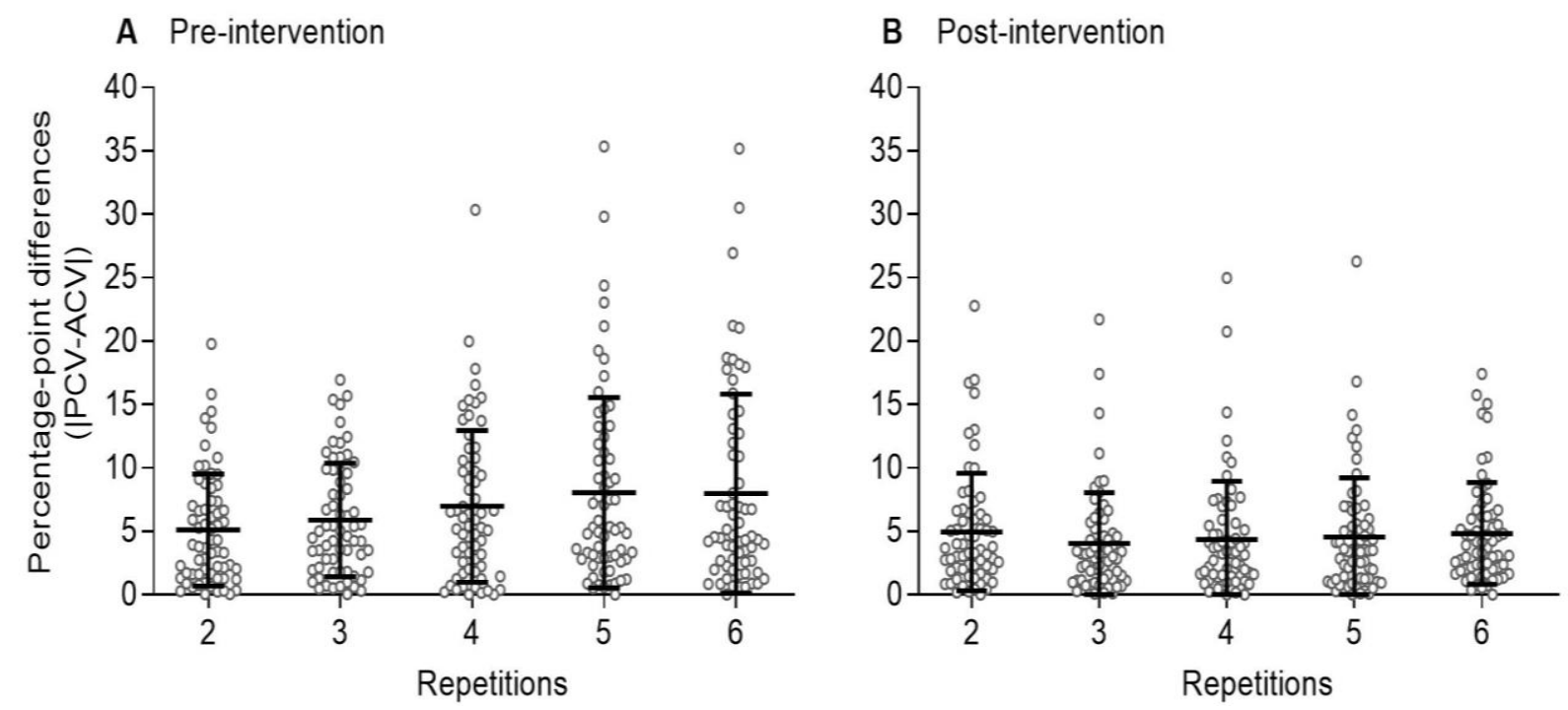

Figure 2. Data points of percentage-point differences between PCV and ACV in absolute value $(|\mathrm{PCV}-\mathrm{ACV}|)$ across sets. The mid-horizontal line and error bars represent means and SDs. 

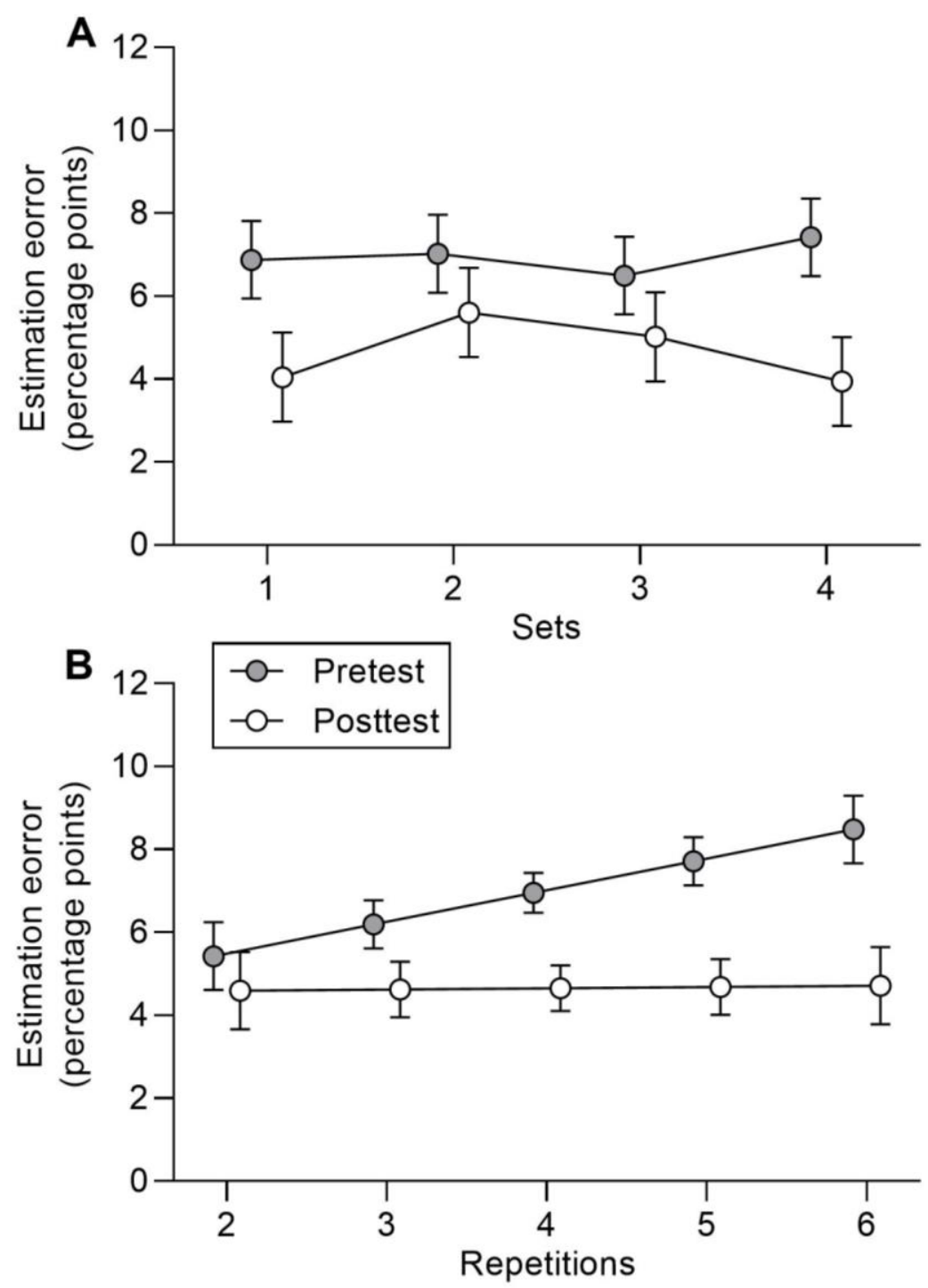

Figure 3. Estimations of errors (|PCV-ACV|) across sessions and sets (A) or repetitions (B), when all other variables are set to their grand mean. Circles and error bars represent estimations and $95 \%$ confidence intervals.

\section{Discussion}

The purpose of this study was twofold. The first, to examine the PCV accuracy levels of athletes experienced with VBT in the barbell squat. The second was to examine if and to what extent can PCV accuracy levels improve among this cohort after a single session of 
verbal and visual feedback on PCV accuracy. During the pre-intervention session, the absolute error rates increased with consecutive repetitions. In the post-intervention session, after receiving feedback on PCV, athletes improved their accuracy ratings. This consisted of a mitigation of the increment in error associated with each subsequent repetition.

The absolute error in the pre-intervention session followed a similar trend to that observed in Sindiani's study, ${ }^{12}$ in which the extent of error grew with consecutive repetitions, although it was smaller in the current study. These findings can be explained by the fact that the athletes in the present study had extensive experience with VBT, in contrast to those in Sindiani et al. ${ }^{12}$ However, other factors could potentially explain these differences such as the loads lifted and number of repetitions completed across the sets. In the current study, athletes completed six repetitions per set using their individual OPL in the squat, which corresponded to $\sim 52 \%$ of estimated 1RM. In contrast, in Sindiani et al study, individuals completed eight repetitions per set using $60 \%$ and $70 \%$ of 1RM. It is possible that the heavier lifted loads coupled with the higher number of repetitions may have led to greater neuromuscular fatigue which could partly explain the greater error observed in Sindiani's study. ${ }^{12}$

Based on the pre-intervention results, we conclude that athletes with VBT experience begin with reasonable PCV accuracy rates although these progressively worsen with subsequent repetitions. From a practical perspective, it is debatable if the observed error rates can be tolerated by coaches, especially in cases when the more conservative (i.e., 5-20\%) velocity loss thresholds are implemented to terminate the training sets. However, PCV may still be used in sessions including velocity loss thresholds of 20-30\% to which PCV error rates of $5 \%$ and $8 \%$, will likely have a marginal impact on the overall training responses.

The post-intervention values indicate that a single session in which verbal and visual feedback were provided on the extent of error considerably improved accuracy rates. More specifically, athletes reduced the absolute error, on average, by 2.3 percentage points from $\sim 7 \%$ in the pre-intervention session to $\sim 4.7 \%$ in the post-intervention session. While augmented feedback is a known strategy to improve accuracy in motor tasks ${ }^{14,15}$, the pattern of improvement observed in this study is interesting and somewhat surprising. Whereas the extent of absolute error of the second repetition was mostly similar to the pre- 
intervention values (pre: 5\%, post: $4.7 \%$ ), the gradual increments in error were eliminated as the error rates remained approximately constant across repetitions (Figure 2). Given that different types of feedback are known to influence motor learning ${ }^{16}$ and estimation abilities ${ }^{17}$ differently, it can be speculated that the provided feedback emphasized error control during sets rather than error reduction of single repetitions. From a practical perspective, the PCV accuracy improvements are encouraging. They indicate that athletes without regular access to velocity tracking devices can improve PCV accuracy after only a single session that includes feedback on the extent of error.

A number of methodological aspects of this study warrant discussion. First, due to COVID19, five athletes did not complete the third and fourth sessions. However, given that these athletes did not differ, significantly or substantially, from the rest of the sample in the extent of error in the pre-intervention session, the attrition does not seem to be related to the dependent variable and thus does not bias the results. Moreover, since we tested a relatively homogenous group of national-level soccer athletes, it is unlikely that attrition bias may have arisen. Second, this study did not include a control group that repeated the same procedure but without receiving any feedback in the third session. The reason we did not include a control group stemmed from our inability to recruit a much larger sample of athletes, but also because our goal was to examine if PCV can be improved, and if so, to what extent. Generating an answer to this question, even absent of the specific causal pathway, was of practical value, and one we were able to answer with the implemented design. It is also possible that additional feedback sessions can reduce the extent of error further, and that providing slightly different types of feedback could reduce the error rates earlier on in a set (e.g., see references ${ }^{16,17}$ ). However, these possibilities need to be determined in future studies. Finally, we only tested a single exercise among a relatively homogeneous sample with VBT experience. Accordingly, these results cannot be generalized to other exercises and populations.

\section{Practical Application}

Coaches who wish to implement VBT methodologies among athletes with VBT experience can do so with reasonable accuracy using athletes PCV as a strategy for set termination. In order to further improve PCV accuracy, it is suggested to have athletes go through at least 
one session in which athletes receive feedback about their errors. This is expected to improve their accuracy rates, mostly as sets progress. We note that this practical suggestion is limited to athletes with VBT experience in the squat exercise, and to coaches who are willing to accept some degree of error regarding the actual velocity at the point of the set termination.

\section{Conclusion}

We observed that athletes with VBT experience in the squat exercise begin with reasonable PCV accuracy rates that worsens as the sets progress. When athletes went through a single session that included augmented feedback about their PCV errors, they substantially improved their accuracy rates in the subsequent session, in which feedback was not provided. The pattern of improvement stemmed from the reduction of the progressive worsening of PCV accuracy rates that were present in the pre-intervention session.

\section{References}

1. Guerriero A, Varalda C, Piacentini MF. The Role of Velocity Based Training in the Strength Periodization for Modern Athletes. J Funct Morphol Kinesiol. 2018;3(4):55-66.

2. Jovanović M, Flanagan EP. Researched applications of velocity based strength training. J Aust Strength Cond. 2014;22(2):58-69.

3. Nevin J. Autoregulated Resistance Training: Does Velocity-Based Training Represent the Future? Strength Cond J. 2019;41(4):34-39.

4. Mann JB, Ivey PA, Sayers SP. Velocity-based training in football. Strength Cond J. 2015;37(6):52-57.

5. Sánchez-Medina L, gonzález-Badillo JJ. Velocity Loss as an Indicator of Neuromuscular Fatigue during Resistance Training. Med Sci Sports Exerc. 2011;43(9):1725-1734.

6. Gonzalez-Badillo JJ, Yanez-Garcia JM, Mora-Custodio R, Rodriguez-Rosell D. Velocity Loss as a Variable for Monitoring Resistance Exercise. Int J Sports Med. 2017;38(3):217-225.

7. Pareja-Blanco F, Sánchez-Medina L, Suárez-Arrones L, González-Badillo JJ. Effects of velocity loss during resistance training on performance in professional soccer players. Int J Sports Physiol Perform. 2017;12(4):512-519.

8. Pareja-Blanco F, Rodríguez-Rosell D, Sánchez-Medina L, et al. Effects of velocity loss during resistance training on athletic performance, strength gains and muscle adaptations. Scand J Med Sci Sports. 2017;27(7):724-735. 
9. Sánchez-Moreno M, Cornejo-Daza PJ, González-Badillo JJ, Pareja-Blanco F. Effects of Velocity Loss During Body Mass Prone-Grip Pull-up Training on Strength and Endurance Performance. J Strength \& Cond Res. 2020;34(4):911917.

10. Pérez-Castilla A, Piepoli A, Delgado-García G, Garrido-Blanca G, García-Ramos A. Reliability and concurrent validity of seven commercially available devices for the assessment of movement velocity at different intensities during the bench press. J Strength \& Cond Res. 2019;33(5):1258-1265.

11. Banyard HG, Nosaka K, Sato K, Haff GG. Validity of Various Methods for Determining Velocity, Force, and Power in the Back Squat. Int J Sports Physiol Perform. 2017;12(9):1170-1176.

12. Sindiani M, Lazarus A, Iacono AD, Halperin I. Perception of changes in bar velocity in resistance training: Accuracy levels within and between exercises. Physiol \& Behav. 2020;224:113025.

13. Loturco I, Nakamura FY, Tricoli V, et al. Determining the optimum power load in jump squat using the mean propulsive velocity. PloS one. 2015;10(10).

14. Brindle TJ, Mizelle J, Lebiedowska MK, Miller JL, Stanhope SJ. Visual and proprioceptive feedback improves knee joint position sense. Knee Sur Sports Traumatol Arthrosc. 2009;17(1):40-47.

15. Moran KA, Murphy C, Marshall B. The need and benefit of augmented feedback on service speed in tennis. Med Sci Sports Exerc. 2012;44(4):754-760.

16. Lauber B, Keller M. Improving motor performance: Selected aspects of augmented feedback in exercise and health. Eur J Sport Sci. 2014;14(1):36-43.

17. Richardson AR, Waller D. The effect of feedback training on distance estimation in virtual environments. J Appl Res Mem Cogn. 2005;19(8):1089-1108. 